the Physics and Astronomy

\title{
Vortex Turbulence in Linear Schroedinger Wave Mechanics
}

\author{
Tzihong Chiueh $*$ Tak-Pong Woo $₫$ Hung-Yu Jian \\ 1. Department of Physics, \\ National Taiwan University, 106, Taipei, Taiwan \\ 2. Center for Theoretical Sciences, \\ National Taiwan University, 106, Taipei, Taiwan \\ 3. Center for Quantum Sciences and Engineering, \\ National Taiwan University, 106, Taipei, Taiwan \\ 4. LeCosPa, National Taiwan University, 106, Taipei, Taiwan \\ 5. Department of Physics, Soochow University
}

(Dated: November 5, 2018)

\begin{abstract}
Quantum turbulence that exhibits vortex creation, annihilation and interactions is demonstrated as an exact solution of the time-dependent, free-particle Schroedinger equation evolved from a smooth random-phased initial condition. Relaxed quantum turbulence in 2D and 3D exhibits universal scaling in the steady-state energy spectrum as $k^{-1}$ in small scales. Due to the lack to dissipation, no evidence of Kolmogorov-type forward energy cascade in 3D or inverse energy cascade in $2 \mathrm{D}$ is found, but the rotational and potential flow components do approach equi-partition in the scaling regime. In addition, the $3 \mathrm{D}$ vortex line-line correlation exhibits universal behavior, scaled as $\Delta r^{-2}$, where $\Delta r$ is the separation between any two vortex line elements, in fully developed turbulence. We also show that quantum vortex is not frozen to the matter, nor is the vortex motion induced by other vortices via Biot-Savart's law. Thus, quantum vortex is actually a nonlinear wave, propagating at a speed very different from a classical vortex.
\end{abstract}

PACS numbers: 67.10.Fj, 47.27.-i, 47.32.-y, 47.50.-d, 05.10.-a, 47.11.-j

\section{INTRODUCTION}

Vortices are probably the most common features in classical fluid turbulence, regardless of compressible or incompressible flows. Likewise, quantized vortex lines and tangles not only are frequently observed but also play vital roles in quantum turbulence $[1,2,3]$. Quantized vortices also exist in type II superconductors, representing defects in superconductivity within which resistivity is finite [4]. Recent interests in quantum vortices have been motivated by the intriguing dynamics in Bose-Einstein Condensation [5.6,7]. Particularly interesting is the existence of Kelvin waves on vortex lines [8,9]. Kelvin waves are helical waves and believed to be responsible for coupling quantum turbulence to dissipation.

While two dimensional quantum vortices can be created or annihilated in pair, three dimensional vortex lines can change their topology through reconnection. These intriguing dynamical behaviors have previously been reported with a simple model [10]. However, it is not clear how quantum turbulence may arise from abundant quantum vortex excitations, with the dynamics of each vortex obeying what is described by that work.

Turbulence can occur when an abundance of free energy is available. External forcing is one option to pro-

\footnotetext{
*Electronic address: chiuehth@phys.ntu.edu.tw

${ }^{\dagger}$ Electronic address: bonwood@scu.edu.tw

${ }^{\ddagger}$ Electronic address: hyj@phys.ntu.edu.tw

$\S$ Electronic address: b88202011@ntu.edu.tw
}

vide the free energy, yielding the driven quantum turbulence. A configuration unstable to large scale fluctuations, such as that due to gravity [11], is an alternative to make the free energy available, creating the relaxed turbulence. Driven turbulence requires an energy sink or dissipation so as to achieve detailed balance in a steady state. Relaxed turbulence on the other hand does not necessarily involve in dissipation. But when it does, turbulence will only be a transient phenomenon and will eventually decay away. On the other hand, dissipationless relaxed turbulence that obeys Hamiltonian dynamics with constant energy is a rather uncommon phenomenon in a macroscopic world, since when all degrees of freedom are excited the smallest scale in real systems can often couple to dissipation. Moreover in most theoretical models of turbulence, integration of dynamical equations must often involves numerical dissipation so as to warrant numerical stability, thereby rendering investigation of dissipationless turbulence difficult.

Dissipationless dynamics can nevertheless exist in isolated microscopic systems. How can a microscopic dynamical system evolve to a turbulence state? A turbulent system is normally conceived to be chaotic in detail but universal (insensitive to initial conditions) on average. It is unquestionable that a many-body system with nonlinear interactions can be chaotic. However, it is unclear how a universal state can dynamically arise from a Hamiltonian system. One of the goals of this work is to demonstrate such a universal non-equilibrium state in a Hamiltonian system.

Another important feature which may also exist in tur- 
bulence is the coherent structure [12]. Coherent structures are comparatively longer-lived than other more common random flows in turbulence, and coherent structures may even possess correlation among different length scales of great disparity. The latter property suggests that local scale coupling, which is the basis of the Kolmogorov turbulence cascade scenario, does not necessarily hold, and can lead to intermittency and deviation from the universal turbulence spectrum derived from the mean-field theory [13]. Quantum vortices may be regarded as such coherent objects existing in quantum turbulence, and we will show that their existence can alter the turbulence small-scale energy spectrum.

Inspired by the concept developed for classical fluid turbulence, we are motivated to examine quantum turbulence in the framework of vortex creation and annihilation. Due to the strong nonlinearity in turbulence we shall in principle investigate this problem numerically. However, numerical simulations can often yield uncontrollable numerical dissipation that depends on the adopted numerical schemes. Because the quantum dynamics obeys certain exact symmetries, such as the unitary condition, which can be difficult to be strictly reinforced in numerical simulations, we therefore study a simplest, soluble but non-trivial case in this work - a system described by the Schroedinger equation of free particle with random-phased initial conditions. In the context of superfluid, our system corresponds to the regime where the vortex healing length diverges.

This system in wavefunction formulation is linear and exactly soluble. However, in the fluid formulation, this linear quantum system can be strongly nonlinear. When compared with the nonlinear Schroedinger equation system, the difference is simply the absence of polytropic fluid pressure in the linear Schroedinger case. The polytropic fluid pressure is responsible for sound emission outside vortex cores and important only on large scale. Due to the existence of the polytropic fluid pressure, in addition to the forward cascade of kinetic energy the occupation number per mode can also exhibit an inverse cascade in favor of large-scale excitation. (In the linear Schroedinger case, the occupation number per mode is conserved and the inverse cascade is prohibited.) But important features of quantum turbulence, such as vortices, are of small scale. It is therefore anticipated that the linear Schroedinger equation should be able to capture these small-scale features.

This reasoning is strengthened by the following observation of duality for the nonlinear Schroedinger system. At the very location of vortex where the fluid formulation becomes strongly nonlinear and the density vanishes, the system becomes linear in the wave formulation. The same argument also applies to Kelvin waves which are located at the low-density vortex cores. By contrast, the strongly nonlinear regime of a nonlinear Schroedinger system in the wave formulation can be approximated in the fluid formulation by a linear equation for sound waves. The duality conjugation of strong cou- pling regimes between these two different formulations leads us to believe that our linear wave model is essential to understand quantum vortices and has generic relevance to quantum turbulence of the full problem described by a nonlinear Schroedinger equation.

This paper is organized as follows. Sec.(II) presents the fluid formulation, an alternative to the conventional wave formulation, and the connections of the two in terms of vortices. We give the numerical results of quantum turbulence prior to full excitation of vortices in Sec.(III). Vortices in fully developed turbulence are addressed in Sec.(IV). Our conclusion is given in Sec.(V). Throughout this work, we let $\hbar=1$ and mass $m=1$.

\section{FLUID FORMULATION}

To show the nonlinearity in the fluid variables of the free-particle Schroedinger equation, we cast the wave equation into a set of fluid equations. Let the complex wavefunction be represented by two real functions, $\Psi=f e^{i S}$, with $f$ representing the real amplitude and $S$ the real phase. We further let the fluid density be $\rho \equiv f^{2}$ and the fluid velocity $\mathbf{v} \equiv \operatorname{Im}\left(\Psi^{*} \nabla \Psi\right) / \rho=\nabla S$. The Schroedinger equation then becomes

$$
\frac{\partial f^{2}}{\partial t}+\nabla \cdot\left(f^{2} \nabla S\right)=0
$$

and

$$
\frac{\partial \nabla S}{\partial t}+\nabla\left[\frac{(\nabla S)^{2}}{2}-\frac{\nabla^{2} f}{2 f}\right]=0 .
$$

The first equation represents the conservation of mass. We also replace the original $\nabla(\partial S / \partial t)$ by $\partial(\nabla S) / \partial t$ in the second equation, representing the Euler equation for fluid momentum.

On the other hand, when $S$ becomes a multi-valued function, vortices appear and rotational flows set in. The rotational flow is induced by vortices via Biot-Savart law. The multi-valued $S$ can appear when $\rho$ locally vanishes and the quantum potential $\nabla^{2} f / 2 f$ diverges. Therefore vortices are always located at regions where the wavefunction vanishes $[10,14]$. Since the wavefunction is complex, this condition demands both real part $R$ and imaginary part $I$ to vanish simultaneously, i.e., $\Psi=\left(\mathbf{x}-\mathbf{x}_{0}\right) \cdot \nabla R+i\left(\mathbf{x}-\mathbf{x}_{0}\right) \cdot \nabla I$. Normally $\nabla R$ and $\nabla I$ are not aligned at $\mathbf{x}=\mathbf{x}_{0}$, and the intersection of the two non-parallel surfaces $R=0$ and $I=0$ forms a line in $3 \mathrm{D}$, the vortex line. In $2 \mathrm{D}$, the intersection is a point vortex.

The local density near the vortex can be expressed as $\rho=\left[\left(\mathbf{x}-\mathbf{x}_{0}\right) \cdot \nabla R\right]^{2}+\left[\left(\mathbf{x}-\mathbf{x}_{0}\right) \cdot \nabla I\right]^{2}$, which vanishes quadratically. Upon diagonalization it becomes $\rho=a^{2}\left(x^{\prime}-x_{0}^{\prime}(t)\right)^{2}+b^{2}\left(y^{\prime}-y_{0}^{\prime}(t)\right)^{2}$ where $a$ and $b$ are constants. Without loss of generality, we can therefore let $\Psi \sim a\left(x-x_{0}(t)\right)+i b\left(y-y_{0}(t)\right)$ in an instantaneous diagonal frame where the primes in the above expression 
is dropped for convenience. From this local expression of wavefunction, we find that

$$
\Psi=\sqrt{a^{2}\left(x-x_{0}\right)^{2}+b^{2}\left(y-y_{0}\right)^{2}} e^{i \phi}
$$

where $S=\phi$ which is the space rotation angle around the vortex line. Thus, isolated vortex is generally quantized with a unit angular momentum. (Vortex quantized with an integer multiple of unit angular momentum is also allowed, but it is much less common to be excited in dynamical evolution.) Near the vortex line the leadingorder velocity

$$
\mathbf{v}=\nabla S=\frac{a b r}{\rho} \hat{\phi},
$$

where $\mathbf{r}=\mathbf{x}-\mathbf{x}_{0}$. The velocity diverges at the vortex and the singular vorticity can be defined as

$$
\omega \equiv \nabla \times \nabla S \equiv \frac{\hat{z}}{\pi r_{c}^{2}} \lim _{c \rightarrow 0} \int_{c} \nabla S \cdot d \mathbf{l}=\delta(r) \hat{z},
$$

where the subscript $c$ represents a circular contour along $\phi$ and $r_{c}$ is the radius of the circle $c$. This definition of curl operation makes the singular vorticity circular symmetric. In fact when the density is not circularly symmetric, the leading order velocity also contains compression $\nabla^{2} S$, which also diverges,

$$
\nabla^{2} S=\frac{a b\left(b^{2}-a^{2}\right) \sin (2 \phi)}{r^{2}\left(a^{2} \cos ^{2}(\phi)+b^{2} \sin ^{2}(\phi)\right)^{2}} .
$$

One can further derive that

$$
S=\tan ^{-1}\left(\frac{b}{a} \tan (\phi)\right),
$$

to the leading order near the vortex, and we can uniquely separate the rotational component $S^{r}$ from the potential component $S^{p}$, with $S^{r}=\phi$ and $S^{p}=$ $\tan ^{-1}[(b / a) \tan (\phi)]-\phi$ at the vortex.

In addition, vortex can move at a finite velocity perpendicular to the vortex line. Since the vortex motion is locally two-dimensional, we shall consider the $2 \mathrm{D}$ case in the following local analysis. The vortex motion can be determined from the density evolution equation, since the vortex is tied to $\rho=0$. Substituting the local expression of $\mathbf{v}$ and $\rho$ into Eq.(4), we find that to the leading order $\nabla \cdot(\rho \mathbf{v})=a b \nabla \cdot(r \hat{\phi})=0$. Let $\rho=\rho_{0}+\delta \rho$ and $\mathbf{v}=\mathbf{v}_{0}+\delta \mathbf{v}$, where $\rho_{0}$ and $\mathbf{v}_{0}$ are the leading order density and velocity given above. We then obtain

$$
\nabla \cdot\left(\rho_{0} \delta \mathbf{v}+\delta \rho \mathbf{v}_{0}\right)=(\mathbf{r} \cdot \nabla R) \nabla^{2} I-(\mathbf{r} \cdot \nabla I) \nabla^{2} R
$$

to the next order. Since this equation is a linear equation in $x$ and $y$, we can regard it as the convection of the density null with a finite velocity $\mathbf{w}$; that is, $\mathbf{w} \cdot \nabla \rho_{0}$, where $\mathbf{w}=d \mathbf{x}_{0}(t) / d t$, describing the instantaneous velocity of a vortex. A straightforward algebra shows that

$$
\mathbf{w}=\frac{\operatorname{Re}\left[\nabla^{2} \Psi \nabla \Psi^{*}\right] \times \hat{z}}{2 \nabla \Psi \times \nabla \Psi^{*} \cdot \hat{z}}
$$

where the gradient $\nabla$ is two-dimensional perpendicular to the local vortex line. Note that the propagation velocity is primarily determined by the Laplacian of wave function. This expression is the same as that obtained in [10] with a somewhat different approach.

With this expression for $\mathbf{w}$, we may inquire whether $\mathbf{w}$ is the same as, or can be reduced from, the material velocity $\delta \mathbf{v}$. This question is important since if it does the vortex will be frozen to the quantum fluid and gets advected as the matter does, much like the classical vortex in an isentropic classical fluid. If it does not, the quantum vortex is then a wave pattern, propagating with its own velocity. As far as we are aware of, this important issue has not been addressed in the literature.

Since the vorticity $\omega(\equiv \nabla \times \mathbf{v})$ is a Dirac $\delta$-function, which has circular symmetry, the zeroth-order velocity, being along the circular direction, can not displace the vortex. That is, $\mathbf{v}_{0} \cdot \nabla \omega=0$. It is the next-order velocity $\delta \mathbf{v}$ that can provide a non-vanishing contribution to vorticity advection. Since the leading-order velocity is singular, the next-order velocity can only be obtained by regularization. Regularization of velocity can be performed by averaging the continuity equation, Eq.(1), over an infinitesimally small disk around the vortex:

$$
\frac{\partial\langle\rho\rangle}{\partial t}+\nabla \cdot\langle\rho \nabla S\rangle=0 .
$$

The continuity equation is used to defined the advection velocity because it ensures that the averaged material the vortex is advected by an effective material velocity. Such an average is equivalent to a local angular average, and the regularized velocity $\overline{\delta \mathbf{v}}$ can be shown to be

$$
\overline{\delta \mathbf{v}} \equiv \frac{\langle\delta(\rho \nabla S)\rangle}{\langle\rho\rangle}=\frac{\operatorname{Im}\left[\nabla \Psi^{*} \cdot\left(\nabla \nabla-\mathbf{I} \nabla^{2} / 2\right) \Psi\right]}{2|\nabla \Psi|^{2}},
$$

after a straightforward algebra. Here the identity matrix $\mathbf{I}$ is two dimensional and so is the gradient $\nabla$. The material velocity $\overline{\delta \mathbf{v}}$ near the vortex is proportional to the quadrupole expansion of the wave function and apparently different from the vortex velocity $\mathbf{w}$ which is proportional to the Laplacian of the wave function. It evidences that the vortex is not frozen to the matter and must therefore be a wave pattern.

Finally, we may want to examine whether the vortex motion arises from the induction velocity generated by other vortices via the Biot-Sarvart law. Note that the induction velocity is incompressible, whereas the material velocity described by Eq.(11) is composed of both compressible and incompressible components and therefore the induction velocity is different from the material velocity. This question is important as it has often been assumed so in the literature. We find that the answer is again negative, as can be illustrated from the following simple case.

Consider a two-dimensional free-particle wave function in cylindrical coordinate $(R, \phi), \quad \Psi=c_{0}-$ $J_{1}(k R) e^{i\left(\phi-k^{2} t / 2\right)}$, where $c_{0}$ is a positive constant, $k^{2} / 2$ 
the energy eigenvalue and $J_{1}$ the first-order Bessel function. The density $|\Psi|^{2}$ is stationary in a counterclockwise rotating frame with an angular velocity $R k^{2} / 2$ around $R=0$. This system contains only two vortices of opposite signs near the first peak of $J_{1}$ when $J_{1}\left(k R_{1}\right)>c_{0}$ and $J_{1}\left(k R_{2}\right)<c_{0}$, where $R_{1}$ and $R_{2}$ are the first and second peaks of $J_{1}$. Since the two density nulls are also stationary in the same rotating frame, the two vortices travel together counter-clockwise with the same angular velocity. If the vortex motion were to be due to the induction of the other vortex, the two should have traveled with a speed depending on their separation. One may change the vortex separation by adjusting $c_{0}$. However the angular velocity of the vortex pair remains the same under such a change, meaning that the vortex motion is not caused by the mutual induction.

The nature of a quantum vortex is therefore very different from a classical vortex of an isentropic classical fluid, for which the fluid dynamics is described by the same mass continuity equation as Eq.(1) and a similar momentum equation as Eq.(2). The classical vortex is frozen to the matter. However, the quantum vortex is a nonlinear wave, whose propagation is controlled by the local Laplacian of wave function. As a consequence, the dynamics of quantum vortex, or that of the rotational velocity component, cannot be described by the fluid formulation given by Eqs.(1) and (2) alone for a potential flow. When vortices appear in a quantum fluid, an additional equation of motion for vortex is needed in the fluid formulation to evolve the whole system.

To trace the origin of this issue, let us return to the momentum equation of the fluid formulation, Eq.(2). We shall prove from the vortex motion that the time derivative and the curl operation acting on the rotational (incompressible) components of velocity, $\nabla S^{r}\left(=\mathbf{v}^{r}\right)$, do not commute. (Note that the velocity field also contains a potential (compressible) component, $\nabla S^{p}$, which is regular and not associated with vortices.) First, we shall show that $\nabla$ and $(\partial / \partial t)$ commute when acting on $S^{r}\left(\equiv \tan ^{-1}\left[\left(y-y_{0}(t)\right) /\left(x-x_{0}(t)\right)\right]\right)$. Let the instantaneous velocity vector $\mathbf{w}\left(=d \mathbf{x}_{0} / d t\right)$ be chosen in the $\mathrm{x}-$ direction, $\mathbf{r}_{0}=0$ and $\phi$ be the angle around $r=0$. The equality

$$
\nabla\left(\partial S^{r} / \partial t\right)=\left(w / r^{2}\right)[\cos (2 \phi) \hat{y}-\sin (2 \phi) \hat{x}]=\partial\left(\nabla S^{r}\right) / \partial t
$$

holds near the vortex, ensuring that $\nabla$ and $\partial / \partial t$ commute. Hence Eq.(2) is a valid equation derived from the Schroedinger equation even in the presence of rotational component. Second, the quantity $\partial S^{r} / \partial t=-\sin (\phi) / r$ is a single-valued function, and therefore $\nabla\left(\partial S^{r} / \partial t\right)$ is curl-free. So will $\partial \nabla S^{r} / \partial t$ be curl-free. Upon taking a curl operation on Eq.(2), both sides are identically zero.

On the other hand, we have the vortex equation of motion

$$
\frac{\partial \nabla \times \nabla S^{r}}{\partial t}=-\mathbf{w}(t) \cdot \nabla\left(\nabla \times \nabla S^{r}\right),
$$

where the vorticity $\nabla \times \nabla S^{r}=\delta\left(\mathbf{r}-\mathbf{r}_{0}(t)\right)$. Combin- ing with the fact that $\nabla \times\left(\partial \nabla S^{r} / \partial t\right)=0$, we have the commutation relation

$$
[\nabla \times, \partial / \partial t] \nabla S^{r}=\mathbf{w} \cdot \nabla\left(\nabla \times \nabla S^{r}\right)
$$

which is non-vanishing. This completes our proof.

From the viewpoint of dynamical evolution, we find that the fluid formulation of Quantum Mechanics, Eqs.(1) and (2), are inadequate when vortices exist in the system. A third equation must be supplemented, with the above commutation relation, Eq.(14), or the vortex equation, Eq.(13). This new equation updates the positions of vortices, and the instantaneous rotational velocity $\nabla S^{r}$ outside the vortex can be determined by the Biot-Savart law of induction. Since the vortex velocity w, Eq.(9), depends also on the potential component of the flow $\nabla S^{p}$, the rotational component $\nabla S^{r}$ is thereby coupled to the potential component. On the other hand, the potential component $\nabla S^{p}$ is coupled to the rotational component through the momentum equation, Eq.(2), since $\nabla S$ in this equation contains both $\nabla S^{r}$ and $\nabla S^{p}$. It thus follows that these two topologically different components of flow are non-trivially coupled.

\section{TIME-DEPENDENT SOLUTIONS OF QUANTUM TURBULENCE}

In order to understand the development of vortex creation and the subsequent evolution in quantum turbulence, we solve the time-dependent solutions of the freeparticle Schrodineger equation in a periodic box of $2048^{2}$ grids for $2 \mathrm{D}$ and of $1024^{3}$ grids for $3 \mathrm{D}$. The solution can be given analytically through the Fourier-mode propagator, and the periodic computational box used here serves only to illustrate the turbulence solutions.

\section{A. Initial Conditions and Potential Flow Turbulence}

We define the potential (compressional) flow in the Fourier space as $\mathbf{v}_{\mathbf{k}}^{p} \equiv \hat{k} \hat{k} \cdot \mathbf{v}_{\mathbf{k}}$. The rotational (incompressible) flow is then defined as $\mathbf{v}^{r} \equiv \mathbf{v}-\mathbf{v}^{p}$. We choose the initial condition such that $\rho=1$ throughout the entire domain and let the phase be randomly distributed only on the large scale. Specifically, the Fourier components of $S$ obey a Gaussian random distribution centering at $|\mathbf{k}|=0$ with the variance $|\Delta \mathbf{k}|$ equal to $20 \times 2 \pi / L$ for $2 \mathrm{D}$ and $10 \times 2 \pi / L$ for $3 \mathrm{D}$, where $L$ is the domain size. Thus the initial flow energy is confined on large scales.

We construct the time-dependent solution of the linear Schroedinger equation by using the propagator in Fourier space. Each Fourier component of mode $\mathbf{k}$ is propagated forward in time by multiplying the phase factor $e^{i t \mathbf{k}^{2} / 2}$ to the initial Fourier mode. The updated wavefunction is then used to construct the fluid variables, $\rho$ and $\mathbf{v}$, for exploration of quantum fluid turbulence. 

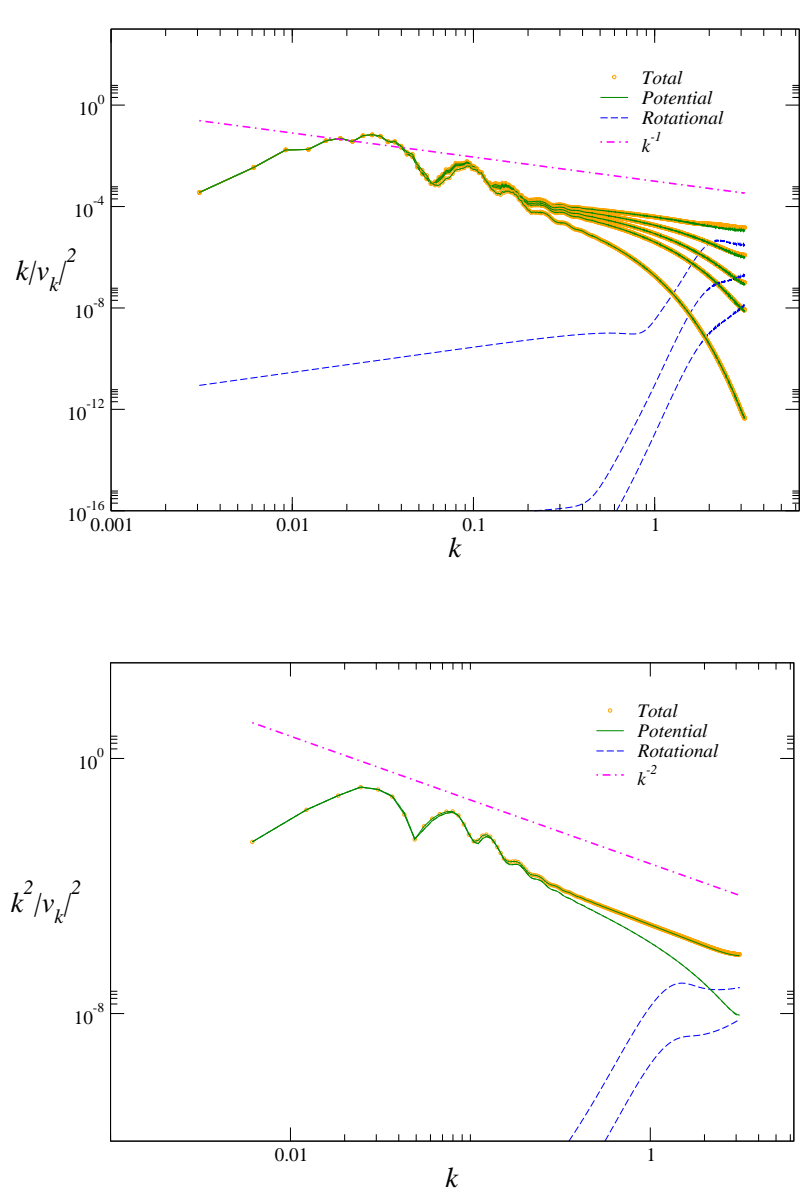

FIG. 1: Evolution of power spectra for potential and rotation flows before the onset of vast excitation of rotational flows for 2D (upper panel) and 3D (lower panel). Cascade of potential flow energy from large scales to small scales is apparent as the small-scale power progressively rises. In the mean time, increasing numbers of vortex pairs are forming during this stage, signified by the small scale excitation in the rotational flow power spectrum.

Due to the nonlinearity of the fluid variable, the flow energy is able to couple from large scales to small scale. At some point in time, the smallest scale features in the computation domain are fully excited by the nonlinear coupling and the vortex flow suddenly appears, as shown in the velocity spectrum (Fig.(1)). It is noted that near the onset of the vortex flow, the small-scale potential flow is fully excited up to the smallest available scale and the spectrum of the potential flow develops a power law behavior, $k^{-1}$ for $2 \mathrm{D}$ and $k^{-2}$ for $3 \mathrm{D}$. This behavior lasts only for a short moment before the rotational flow begins to get excited.

We shall pause here and offer an explanation for the power-law spectrum of the potential flow. In a quantum system studied here, the only dimensional constant is the Planck constant, which has dimension (length)(velocity). It then follows that the velocity difference $|\Delta \mathbf{v}|$ of the potential flow over a distance $|\Delta \mathbf{r}|$ scales as $|\Delta \mathbf{r}|^{-1}$, as a result of uncertainty principle. The Fourier component $\left|\Delta \mathbf{v}_{k}\right| \sim k^{-1}$ for $2 \mathrm{D}$ and $\left|\Delta \mathbf{v}_{k}\right| \sim k^{-2}$ for 3D, yielding a spectral density $\sim k^{-1}$ for $2 \mathrm{D}$ and $k^{-2}$ for $3 \mathrm{D}$. Note that the development of this power-law behavior is through cascades, where modal energy is transferred from large scales to small scales by local coupling in $k$ space. This nonlinear coupling is similar to cascades in classical fluid turbulence.

To gain a better picture of what is going on at the onset of vortex creation, we shall turn to the real-space flow. Figure (2) shows the local rise of $2 \mathrm{D}$ potential-flow velocity due to a large local gradient in quantum potential, corresponding to the situation where $R=0$ and $I=0$ surfaces are locally tangent to each other. Shortly after, two streams of counter-rotating flow develop about the two sides of the peak potential flow velocity to form a tightly bound vortex pair, corresponding to the critical crossing of the two surfaces. The two counter-rotating flow streams grow in strength until their circulations are quantized before the two vortices can separate from each other to become isolated vortices. In $3 \mathrm{D}$, vortex creation is through formation of a vortex loop of infinitesimal radius. The physical picture is similar to what happens for a vortex pair in 2D. Not until the rotational velocity grows to the strength where the circulation is quantized can the vortex loop expand to a finite radius.

\section{B. Vortex Turbulence}

Once created, vortices of opposite signs can move away from each other in 2D and vortex loops can expand in 3D. Starting from our relatively smooth initial condition, after the first vortex pair is created, many more vortex pairs can soon be generated, tapping the free energy in the potential flow. In Figs.(3) and (4), we show a snapshot of point vortices and vortex lines in our $2 \mathrm{D}$ and $3 \mathrm{D}$ computation domains.

The turbulence spectrum long after the onset of vortex fluctuations is shown in Fig.(5). The flow eventually is populated with vortex fluctuations and the spectrum approaches a steady state that also largely obeys a power law as $k^{-1}$ in both 2D and 3D cases. (The high- $k$ upturn in the spectrum is an artifact of the finite-size grid and will be addressed later.) Despite the potential flow turbulence is suppressed in small scale after the rotational flow is vigorously excited, the potential and rotational flow turbulence energies are actually in equi-partition. Any vector field can be decomposed to the potential component and rotational component as $\nabla \phi$ and $\hat{z} \times \nabla \psi$, respectively, in $2 \mathrm{D}$, but as $\nabla \phi$ and $\mathbf{A}$, constrained by $\nabla \cdot \mathbf{A}=0$, in $3 \mathrm{D}$. That is, a $2 \mathrm{D}$ flow has two degrees of freedom arising from the independent $\phi$ and $\psi$, and a $3 \mathrm{D}$ flow has 3 degrees of freedom given by one $\phi$ and and two independent components of $\mathbf{A}$. If these components are non-correlated, equi-partition between potential flow turbulence and rotational flow turbulence is naturally expected. In $2 \mathrm{D}$, the equi-partitioned ratio of potential flow 

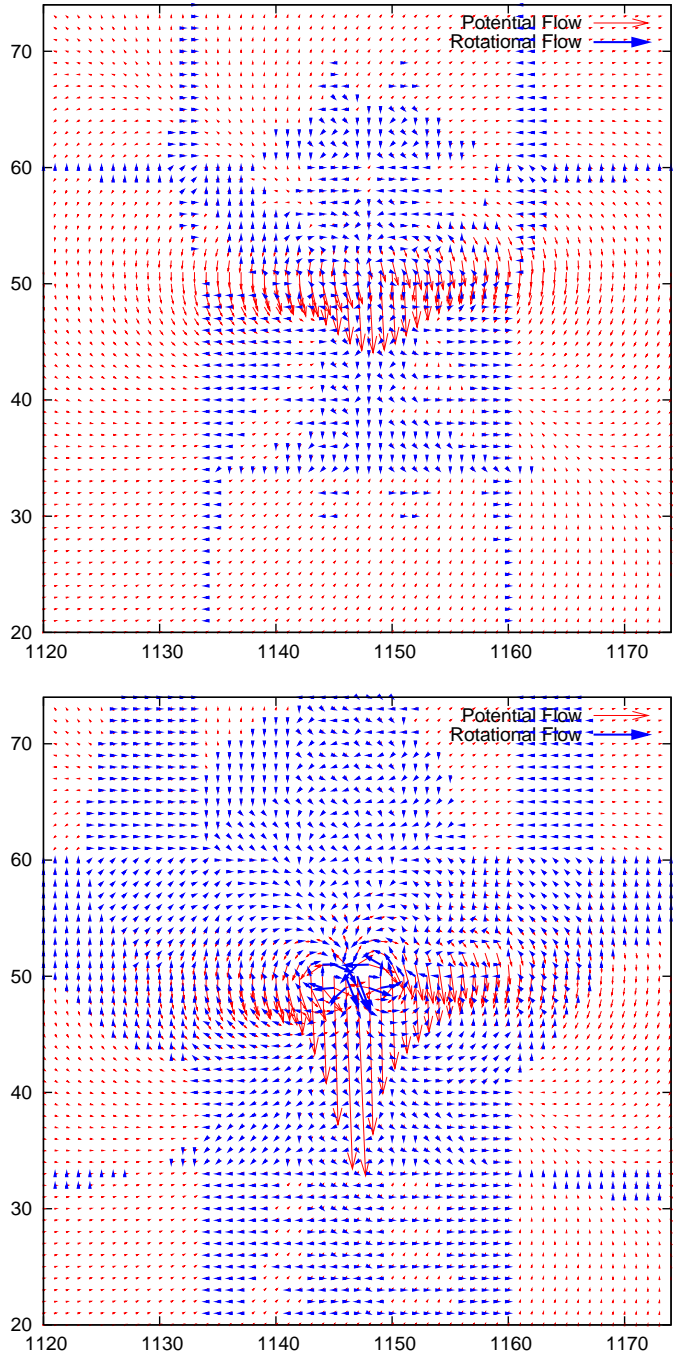

FIG. 2: The real-space flow configuration during vortex-pair creation in 2D. Upper panel shows the sole existence of potential flow (red) prior to the pair creation, where the potential flow locally develops a large velocity gradient. Lower panel shows the birth of a vortex pair, where counter-rotating flows (blue) grow in strength. Not until the rotational flow velocity grows to the magnitude where the circulation is quantized can the pair separate as isolated vortices.

energy to rotational flow energy is 1 , and in $3 \mathrm{D}$ the ratio becomes $1 / 2$. These behaviors are what have been observed in our $2 \mathrm{D}$ and $3 \mathrm{D}$ cases.

Return to the turbulence spectrum. We note that vortices are line objects when viewed in $3 \mathrm{D}$. The fluid velocity is dominated by local $2 \mathrm{D}$ flows around the vortex lines. Therefore when the vortex lines are sparsely distributed in space, the vortex flow velocity has strong short-distance correlation transverse to the line but longdistance correlation along the line, leading to similar 2D

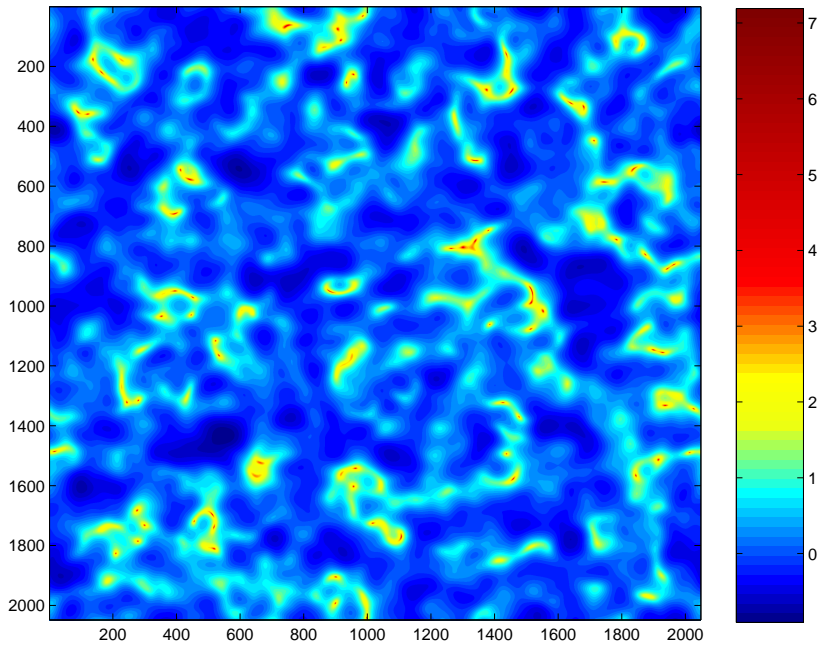

FIG. 3: Real-space image of point vortices in steady-state 2D turbulence. These point vortices are captured by plotting the inverse density of extremely large value. The color code is in logarithmic scale.

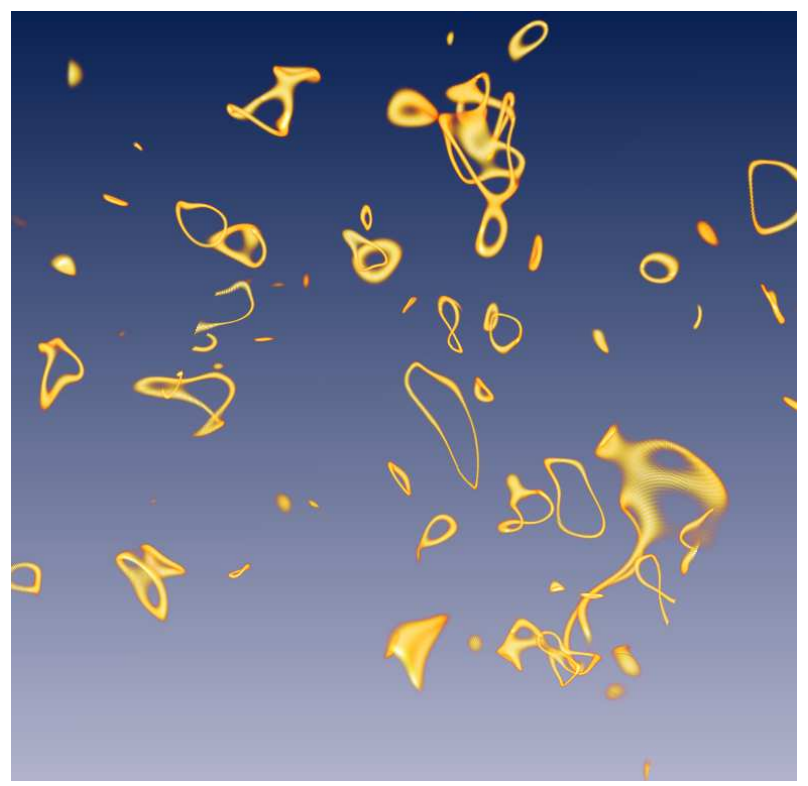

FIG. 4: Real-space image of tangled vortex tubes in steadystate 3D turbulence. These vortex tubes are captured by plotting the constant-density surfaces of very low density.

and $3 \mathrm{D}$ velocity spectra in high $k$. In fact this $k^{-1}$ spectrum may be derived from the spectrum of a single vortex. The transverse velocity around a vortex $\left|\mathbf{v}_{\perp}\right|$ scales as $|\mathbf{r}|^{-1}$. Hence its Fourier component scales as $k^{-1}$, and the spectrum as $k^{-1}$. In reality, there are a number of point vortices and vortex loops in $2 \mathrm{D}$ and $3 \mathrm{D}$. (See 

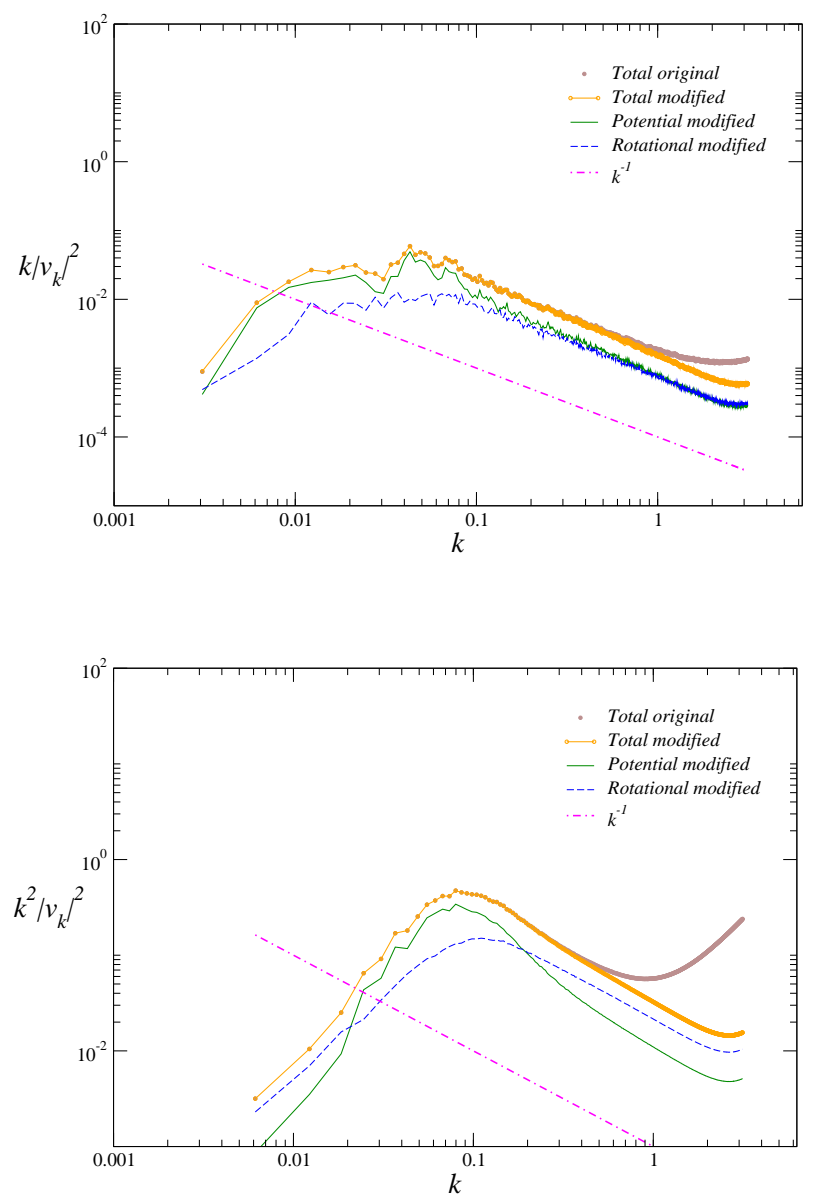

FIG. 5: Steady-state power spectra of 2D (upper panel) and $3 \mathrm{D}$ (lower panel) turbulence. The total flow, potential flow and rotational flow spectra are plotted separately. The original spectra have unphysical upturns at the highest wavenumber due to the grid-size singular flow velocity of vortex that cannot be faithfully captured by the grids. Upon removal of these unphysical pixels, the spectrum shows the almost perfect $k^{-1}$ power law in both $2 \mathrm{D}$ and $3 \mathrm{D}$ turbulence. They also exhibit energy equi-partition on small scales.

Figs.(3) and (4) for real-space 2D and 3D images of vortices.) If these vortices are weakly correlated, the total spectrum is simply the sum of all individual vortex spectra and hence will still obey the $k^{-1}$ power law. Indeed, since the $2 \mathrm{D}$ point vortex occupies a $2 \mathrm{D}$ area of measure zero, so does the 3D loop vortex a zero volume, the vortex-vortex two-point correlation function is therefore overwhelmingly dominated by the zero-separation contribution, and the resulting spectrum remains to be $k^{-1}$.

Nevertheless, the finite-separation contribution to the vortex-vortex two-point correlation should still exist in this system. It is simply due to the strong zero-separation correlation that the finite-separation correlation becomes not obvious. For example, the 2D vortex pair should have kept some memory of its counterpart after pair separation, and therefore they should be correlated. In 3D the vortex-loop element should also be correlated with other elements in the same loop. Correlation of different loops, once exists, should be an indication of non-trivial dynamics in action.

To uncover this weak finite-separation correlation, we treat every point vortex in $2 \mathrm{D}$ as either a signed point or an unsigned point and examine the two-point correlation function of these points. It is found that for the unsigned case, the vortex correlation $\xi(\Delta r)$ does not show any power-law relation with $\Delta r$, where $\Delta r$ is the separation of two points. For the signed case, the two-point correlation $\eta(\Delta r)$ does however exhibit the screening effect, where at small separation the correlation is negative and finite but the correlation abruptly drops to zero at some critical separation. In fact the signed vortex correlation can be fit by a Gaussian. The critical separation, or the variance of the Gaussian, represents the mean vortex separation, which is determined by the initial flow energy with larger flow energy exciting more vortices and therefore yielding smaller vortex mean separation. Figure (6) shows such 2D two-point correlation of signed and unsigned cases.

For 3D finite-separation correlation, we also consider the vortex line as either a directional line or a nondirectional line. The directional line correlation is defined as $\eta(\Delta r) \equiv\left\langle d \mathbf{l}_{1}(\mathbf{r}) \cdot d \mathbf{l}_{2}(\mathbf{r}+\boldsymbol{\Delta} \mathbf{r})\right\rangle$, and the nondirectional line correlation as $\xi(\Delta r) \equiv\left\langle d l_{1}(\mathbf{r}) d l_{2}(\mathbf{r}+\boldsymbol{\Delta} \mathbf{r})\right\rangle$. It turns out that the nondirectional line-line correlation $\xi$ obeys a universal $\Delta r^{-2}$ power law. Although the directional line-line correlation $\eta$ also obeys $\Delta r^{-2}$ power law at small separation, it abruptly drops to zero at a finite separation. The directional line-line correlation $\eta$ reveals the average size of the vortex loop at the separation when the correlation drops to zero. Figure (7) depicts the 3D line-line correlation. Comparing these correlation functions in $2 \mathrm{D}$ and $3 \mathrm{D}$ cases, we find that the dimensionality plays a role in the final states. Two-dimensional quantum turbulence in our model appears farther from a relaxed state than the $3 \mathrm{D}$ case since small-scale mixing in $3 \mathrm{D}$ turbulence is well in place in the final steady state that yields a power-law correlation.

We now turn to the upturn at the highest- $k$ in the energy spectrum. It is actually caused by numerical errors in the finest grid at the vortex. The velocity field is singular at the vortex, whose exact location can be anywhere within the finest grid, giving unequal weights to the grids immediately surrounding the vortex. When the vortex is much closer to a particular grid, the velocity at that grid will be much larger than other nearby grids. Therefore, this grid will appear as an erroneous Dirac $\delta$ function, thereby giving an upturn with the first power of $k$ in the spectrum at the smallest scale. We suppress this problem by limiting the magnitude of velocity to an appropriate upper bound so as to eliminate the contribution of the said $\delta$-functions. (Only 20-30 grids are processed among a total of $2048^{2}$ grids that contains about 100 point vortices in the $2 \mathrm{D}$ case, and the $3 \mathrm{D}$ case also has a similar ratio of processed to unprocessed grids.) Fig- 


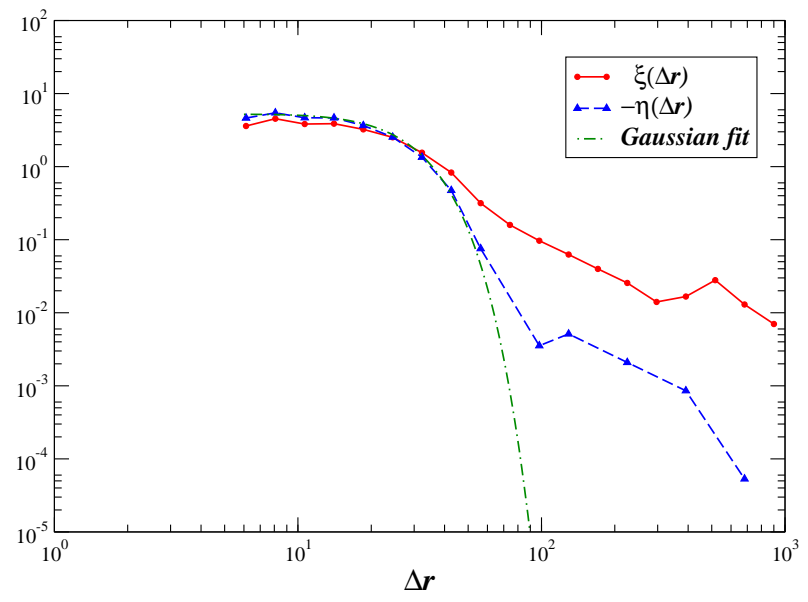

FIG. 6: Two-point correlation functions of signed point vortices $\eta$ and unsigned point vortices $\xi$. The signed vortex correlation $\eta$ can be fit by a Gaussian and the variance of the Gauscian renresents the vortex mean senaration

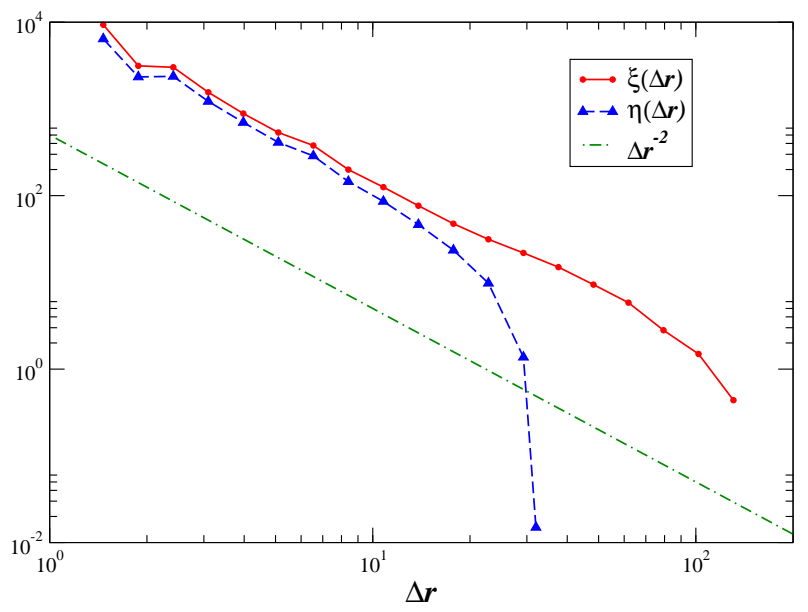

FIG. 7: Line-Line correlation of directional vortex lines $\eta$ and non-directional cortex lines $\xi$. Both follow a power law $\Delta r^{-2}$ at small separation.

ure (5) also shows the spectra after this regularization procedure. We emphasize that such a grid-scale error is unavoidable since the local physics is singular; such an error can accumulate over time when evolving a nonlinear schroedinger equation numerically. Only in this soluble model adopted here can the error in the solution be controllable and does not accumulate.

Quantum vortices are long-lived coherent structures in dissipationless turbulence. Once created, they can only come to destruction through pair annihilation in 2D and through shrinkage of ring vortex in 3D. When vortex lines are sparsely distributed in space, vortex collisions are rare events, thereby making them long-lived objects.
Vortex-line reconnection arises in 3D from vortex collision and is a more common phenomenon than vortex annihilation. Details of 3D vortex-line reconnection are depicted in Fig.(8). Also clear in Fig.(8) is the existence of helical interference fringes along the cores of $3 \mathrm{D}$ vortex lines. These fringes appear to vary rather slowly and are the $m$ (mode number around the tube) $=2$ density fluctuations of varying $n$ (mode number along the tube). We mentioned previously that the density profile cut across a vortex-tube is generally elliptical near the null, and this is consistent with the $m=2$ density fluctuation observed at the $3 \mathrm{D}$ vortex core. We also note that Kelvin waves are the $m=1$ fluctuations and oscillate due to the "tension" of a vortex line. Thus, Kelvin waves appear qualitatively different from these $m=2$ fringes.

In $2 \mathrm{D}$ quantum turbulence, inverse cascade has not been observed in our system. We explore this issue by having the energy of initial potential flow concentrating on the mid scale, hoping to provide an ample space on the large scale for inverse cascades to take place. However, the large-scale flow energy in fact does not change throughout the evolution, indicating the non-existence of inverse cascade in this model. Treating unsigned vortices as individual points, we again examine the vortex-vortex correlation, aiming to find out whether these points have any tendency to cluster when these point vortices are created on an intermediate scale. Like the situation explored previously, we find that $2 \mathrm{D}$ vortices do not develop long-range correlation. This aspect is in great contrast to classical 2D fluid turbulence, where vortices of the same sign tend to aggregate to form large vortex clusters.

\section{CONCLUSIONS}

By exploring the exactly soluble Schroedinger equation for free-particles, we have derived the following generic results in this work. These results can also, or has been observed to, arise from a nonlinear Schroedinger system. (1) Quantum vortex has a axisymmetric rotational velocity but a non-axisymmetric potential velocity, both of which diverge near the vortex as $r^{-1}$. (2) Whenever vortices appear in the system, the density and momentum equations of the fluid representation will become inadequate to evolve the system, and it requires a third equation, the vortex equation of motion, for the full description of evolution. (3) Unlike classical vortex, quantum vortex is a wave that is not frozen into the matter and it propagates at a velocity different from the local matter velocity. (4) The motion of quantum vortex is not entirely induced by other vortices and hence its propagation velocity is different from the rotational component of local matter velocity. (5) Equip-partition between rotational flow energy and potential flow energy can be established on small scales in fully developed quantum turbulence.

Specific results for our 2D and 3D free-particle models are that (a) starting with an initial potential flow, 

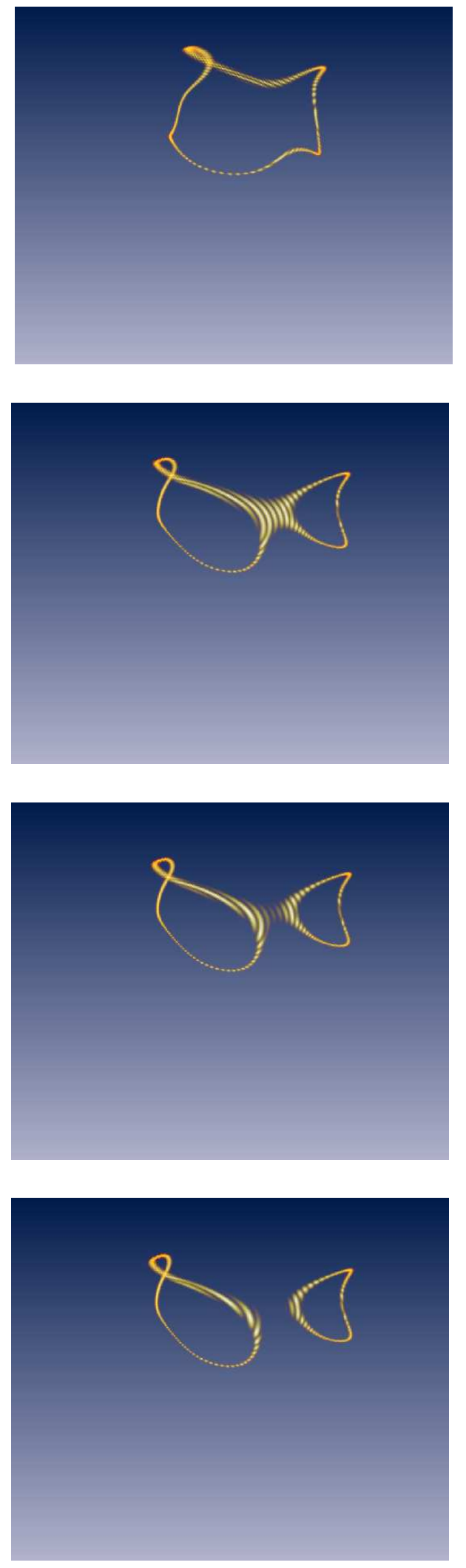

FIG. 8: 3D reconnection of vortex loop, which is the major activity for the maintenance of steady-state quantum turbulence. The evolution proceeds from the upper to bottom panels. We also note the small-scale helical fringes in existence along the cores of vortex lines. These helical fringes appear at the very core of a vortex tube, as they can only be captured when the constant-density surfaces assume an extremely low value, lower than that used to plot Fig.4. turbulence cascades toward small-scale is observed prior to rotational flows are excited, and near the transition to the excitation of rotational flow the energy spectra of potential flow turbulence in $2 \mathrm{D}$ and $3 \mathrm{D}$ obey different power laws; (b) the energy spectra of fully developed $2 \mathrm{D}$ and $3 \mathrm{D}$ turbulence with vortex excitation both obey the same $k^{-1}$ power law, which corresponds to the power spectrum of a single vortex; (c) $\Delta r^{-2}$ power-law vortexvortex correlation is observed only in $3 \mathrm{D}$ but not in $2 \mathrm{D}$ turbulence; (d) there is no evidence of inverse cascade in $2 \mathrm{D}$ turbulence; (e) there is no evidence of Kolmogorov energy cascade in either 2D or 3D turbulence.

By comparing our general and specific results, it is evident that small-scale features derived from this linear Schroedinger equation can be in common with those derived from a nonlinear Schroedinger equation. But the large-scale features, such as vortex-vortex correlation, cannot, because the wave nonlinearity and dissipation, which are lacking in this linear model, must play an essential role in shaping the global dynamics. Along this line, we note that the steady-state turbulence spectrum is maintained not by the conventional cascade processes in this model, since no dissipation is involved. In fact, energy cascade is only observed in the initial potential flow phase as a transient before vortices are excited. Once vortices are fully excited, the steady-state $k^{-1}$ energy spectrum found in this model only reveals the spectrum of a collection of individual vortices and contains no information about correlation among different vortices.

Whether it is the dissipation or the wave nonlinearity that is the prime factor for producing the long-range vortex-vortex correlation is an important issue for understanding the actual quantum turbulence. In fact, in the presence of small-scale dissipation the $k^{-1}$ steady-state spectrum must fail, as it would have yielded a diverging dissipation rate. Whether the Kolmogorov spectrum may be established in a dissipative linear Schroedinger system remains to be investigated. In the case of a nonlinear Schroedinger system, Kelvin wave cascades are found to play a central role in coupling to dissipation $[8,9]$. On this particular issue, our observation for a vortex loop of a linear Schroedinger system is that it can undergo largeamplitude loop distortion so that the loop is self pinched and reconnects to form two smaller loops (c.f., Fig. (8)). This reconnection process can couple to dissipation efficiently if some small scale dissipation is included in the system. On the other hand, despite Kevin waves also ride on a vortex loop and can give rise to large-amplitude loop distortion, the picture of Kelvin wave cascade from long waves to short waves and finally to dissipation on a vortex loop is quite different from our observation of vortex loop dynamics of a linear Schroedinger system.

Finally, we should mention that the present dissipationless linear system in a periodic box can be recurrent in a finite time when the longest wave in the system oscillates over one period. The steady state turbulence presented in this work is found to be already established within a small fraction $(10 \%)$ of the recurrent time. The 
Poincare recurrence time of this model is proportional to the square of the periodic box size, $L^{2}$. Despite the dynamics of this system may appear chaotic, it is not quite a chaotic system due to the algebraic, instead of an exponential, dependence of the recurrence time on the degrees of freedom.

\section{Acknowledgments}

This work is supported in part by the grant, NSC972628-M002-008-MY3, from National Science Council of Taiwan.

[1] R. P. Feynman, Progress in Low Temperature Physics (North-Holland, Amsterdam, 1955), Vol. I.

[2] J. Yepez, G.Vahala, L. Vahala,3 and M. Soe, Phys. Rev. Lett. 103, 084501 (2009); C. Nore, M. Abid, and M. E. Brachet, Phys. Rev. Lett. 78, 3896 (1997); C. F. Barenghi, Physica (Amsterdam) 237D, 2195 (2008); E. Kozik and B. Svistunov, Phys. Rev. Lett. 92, 035301 (2004); V. S. L'Vov, S.V. Nazarenko, and O. Rudenko,
Phys. Rev. B 76, 024520 (2007); W. F. Vinen, M. Tsubota, and A. Mitani, Phys. Rev. Lett. 91, 135301 (2003); E. Kozik and B. Svistunov, Phys. Rev. B 77, 060502(R) (2008)

[3] T. Chiueh, Phys. Rev. E 57, 4150 (1998)

[4] A.A. Abrikosov, Rev. Mod. Phys. 76, 975 (2004)

[5] M.R. Matthews, et al., Phys. Rev. Lett. 83, 2498 (1999)

[6] C.N. Weiler, et al., Nature, 455, 948 (2008)

[7] D.V. Freilich, et al., Science, 329, 1182 (2010)

[8] W.F. Vinen, J. Low Temp. Phys. 145, 7 (2006)

[9] M. Kobayashi and M. Tsubota, Phys. Rev. Lett. 94, 065302 (2005); M. Kobayashi and M. Tsubota, J. Phys. Soc. Jpn. 74, 3248 (2005); C. F. Barenghi, Physica (Amsterdam) 237D, 2195 (2008); T. P. Simula, T. Mizushima, and K. Machida, Phys. Rev. Lett. 101, 020402 (2008); A.L. Fetter, 2004 Phys. Rev. A, 69, 043617 (2004); V. Bretin, P. Rosenbusch, F. Chevy, G.V Shlyapnikov, and J. Dalibard, Phys. Rev. Lett., 90,100403 (2003)

[10] I. Bialynicki-Birula, Z. Bialynicka-Birula, and C. Sliwa1, Phys.Rev. A, 61, 032110 (2000)

[11] T.P. Woo and T. Chiueh, ApJ, 697, 850 (2009)

[12] M. A. Green, C. W. Rowley, and G. Haller, J Fluid Mech., 572, 111 (2007)

[13] Z.S. She and E. Leveque, Phys. Rev. Lett. 72, 336 (1994); Z.S. She and E.C. Waymire, Phys. Rev. Lett, 74, 262 (1995); D. Berengere, Phys. Rev. Lett. 73, 959 (1994)

[14] R. J. Donnelly, Quantized Vortices in Helium II (Cambridge University Press, Cambridge, England, 1991) 